\title{
Desarrollo del pensamiento crítico en estudiantes universitarios
}

\author{
Development of critical thinking in university students
}

Desenvolvimento do pensamento crítico em estudantes universitários

\section{ARTÍCULO GENERAL}

Benites Seguín, Lucio Alfredo

https://orcid.org/0000-0002-4392-1403

lbenitess@ucvvirtual.edu.pe

Universidad César Vallejo, Lima - Peru
Henry Camizán García

Damián Ponte, Isaías Francisco

https://orcid.org/0000-0002-0350-9078

hcamizan@ucvvirtual.edu.pe

Universidad César Vallejo, Lima - Peru https://orcid.org/0000-0002-1005-2109

idamianp@ucvvirtual.edu.pe

Universidad César Vallejo, Lima - Peru

Recibido 08 de Noviembre 2020 | Arbitrado y aceptado 28 de Noviembre 2020 | Publicado en 05 Marzo 2021

\section{RESUMEN}

El objetivo de este artículo es examinar la situación del Estado del Arte respecto al del desarrollo del pensamiento crítico en estudiantes universitarios; para ello, se han examinado séis trabajos de investigación, artículos y tesis, nacionales e internacionales. El criterio de inclusión es el trabajo de investigación referido a universidades y de manera especial en Ciencias Sociales. Se excluyen a todas las demás investigaciones que no tengan esta característica. Como conclusión podemos mencionar, luego de examinar las investigaciones mencionadas existen coincidencias en todas ellas. Todas han utilizado el enfoque cuantitativo y han aplicado instrumentos específicos para extraer datos y realizar la hermenéutica especifica. Asimismo, en todas las conclusiones se puede observar que las estrategias utilizadas producen un significativo desarrollo del pensamiento crítico. Respecto a las diferencias se ha podido indentificar que Lara, V., Ávila, J. y Olivares, S. (2010) ha utilizado un enfoque mixto utilizando un instrumento cuantitativo tipo cuestionario $y$ un instrumento cualitativo tipo rúbrica. Otra diferencia significativa presenta Azurín, V. (2018), utilizando em método cuasiexperimental en su investigación; separó a los estudiantes en dos grupos, uno experimental y otro de control. Así, se ha podido examinar el Estado del Arte respecto al desarrollo del pensamiento crítico.

Palabras Claves : Pensamiento crítico, dimensiones, metodología

\section{ABSTRACT}

The objective of this article is to examine the state of the art situation regarding the development of critical thinking in university students; For this, six national and international research papers, articles and theses have been examined. The inclusion criterion is the research work related to universities and especially in Social Sciences. All other investigations that do not have this characteristic are excluded. As a conclusion we can mention, after examining the aforementioned investigations, there are coincidences in all of them. All have used the quantitative approach and have applied specific instruments to extract data and perform specific hermeneutics. Likewise, in all the conclusions it can be observed that the strategies used produce a significant development of critical thinking. Regarding the differences, it has been possible to identify that Lara, V., Ávila, J. and Olivares, S. (2010) have used a mixed approach using a questionnaire-type quantitative instrument and a rubric-type qualitative instrument. Another significant difference is presented by Azurín, V. (2018), using the quasi-experimental method in his research; He separated the students into two groups, one experimental and one control. Thus, it has been possible to examine the State of the Art regarding the development of critical thinking.

Key Words: Critical thinking, dimensions, methodology

\section{RESUMO}

O objetivo deste artigo é examinar o estado da arte em relação ao desenvolvimento do pensamento crítico em estudantes universitários; Para tanto, foram examinados seis trabalhos de pesquisa, artigos $\mathrm{e}$ teses nacionais $\mathrm{e}$ internacionais. $\mathrm{O}$ critério de inclusão são os trabalhos de pesquisa relacionados às universidades e principalmente nas Ciências Sociais. Todas as outras investigações que não possuem essa característica são excluídas. Como conclusão podemos citar que, após o exame das referidas investigações, há coincidências em todas elas. Todos utilizaram a abordagem quantitativa $\mathrm{e}$ aplicaram instrumentos específicos para extrair dados e realizar hermenêuticas especificas. Da mesma forma, em todas as conclusões pode-se observar que as estratégias utilizadas produzem um desenvolvimento significativo do pensamento crítico. Em relação às diferenças, foi possivel identificar que Lara, V., Ávila, J. e Olivares, S. (2010) utilizaram uma abordagem mista utilizando um instrumento quantitativo do tipo questionário e um instrumento qualitativo do tipo rubrica. Outra diferença significativa é apresentada por Azurín, V. (2018), utilizando o método quase-experimental em suas pesquisas; Ele separou os alunos em dois grupos, um experimental e um controle. Assim, foi possivel examinar o estado da arte quanto ao desenvolvimento do pensamento crítico.

Palavras-chave: Pensamento crítico, dimensões, metodologia 
Introducción

Desarrollar el pensamiento crítico es una necesidad para toda persona, más aún si se dedica al estudio de las Ciencias Sociales; en ese sentido, deberá entenderse que uno de los objetivos educativos primordiales será desarrollar esta capacidad intelectual (Vallarini, 2003). Ya desde la antigüedad con Sócrates (V a.C.), pasando por Descartes (XVII) y llegando hasta Jhon Dewey (XX), se ha tratado de dar énfasis al desarrollo del pensamiento crítico en el razonamiento humano. Ahora bien; cuando se intenta desarrollar el pensamiento crítico en Ciencias Sociales en estudiantes de todos los niveles educativos, nos enfrentamos a un gran dilema, ¿Qué dimensiones, de la diversidad que existen, serán las más adecuadas?

El ser humano desarrollará pensamiento crítico en la medida que examine su pensamiento bajo cinco dimensiones: lógica, que le da claridad y coherencia; sustantiva, porque la información recibida debe corresponder a determinada epistemología; contextual, porque debe tener sentido social actual; dialógica, en la medida que el pensante asuma proponga y asuma las consecuencias de su pensamiento; y pragmática, si al hacerse un examen del pensamiento, este responda al interés que se esté buscando o necesitando. Considerando así el pensamiento crítico y entendiendo que está condicionado por los actores culturales, podemos aseverar que el pensamiento del ser humano no es autónomo y está íntimamente relacionado a su desarrollo moral (Villarini, 2003). Mahmood (2017) identifica 4 sub variables. Al investigar sobre la forma como se identifica el nivel del pensamiento crítico se aplican estándares universales, entre ellos: Claridad, veracidad, precisión, relevancia, profundidad, amplitud y lógica (Paul, R. y Elder, 2005)

El objetivo de la presente investigación es entonces examinar la situación del Estado del Arte respecto al desarrollo del pensamiento crítico en estudiantes universitarios.

\section{Método}

Para realizar esta investigación se han revisado artículos que tienen como tema principal el uso de dimensiones del pensamiento crítico; estos artículos corresponden a universidades de nuestro país Perú, como también algunos países tales como Ecuador, Chile, España, México y Colombia, los mismos que presentaremos más adelante. Esta información ha sido extraída de base de datos tales como: Google Scholar, La Referencia, Scielo, repositorios de universidades como UCV, Unión, etc. De los trabajos de investigación referidos se han seleccionado aquellos que tienen relación con el 
pensamiento crítico en Ciencias Sociales. En una matriz, se presentan los trabajos de investigación estudiados considerando cada metodología usada y participantes del estudio; luego realizamos una comparación de resultados y conclusiones. Los criterios de inclusión son: Que sean artículos científicos o tesis de doctorado, año de publicación a partir del 2010 y que hayan utilizado en su investigación dimensiones de la variable pensamiento crítico. La variable específica para realizar la búsqueda de información ha sido pensamiento crítico y dimensiones del pensamiento crítico

Cuadro 1. Trabajos de investigación estudiados

\begin{tabular}{|c|c|c|c|c|c|}
\hline $\mathrm{N}^{\circ}$ & AIS & AÑO & AUTOR(ES) & METODOLOGÍA & PARTICIPANTES \\
\hline 01 & Colombia & 2010 & $\begin{array}{l}\text { Verónica LARA QUINTERO } \\
\text { José Enrique AVILA PALET } \\
\text { Silvia Lizett OLIVARES } \\
\text { OLIVARES }\end{array}$ & Cuantitativo & $\begin{array}{l}50 \text { estudiantes de In- } \\
\text { geniería Biomédica de una } \\
\text { universidad privada de la } \\
\text { ciudad de Bogotá. }\end{array}$ \\
\hline 02 & Perú & 2018 & $\begin{array}{l}\text { Vilma Urbana AZURÍN } \\
\text { CASTILLO }\end{array}$ & $\begin{array}{l}\text { Cuantitativo } \quad \text {-cuasi } \\
\text { experimental }\end{array}$ & $\begin{array}{l}\text { Estudiantes del IV Ciclo de } \\
\text { la Facultad de Ciencias } \\
\text { Sociales y Humanidades de } \\
\text { la Universidad Nacional de } \\
\text { Educación Enrique } \\
\text { Guzmán y Valle, en el año } \\
2015\end{array}$ \\
\hline 03 & Ecuador & 2018 & $\begin{array}{l}\text { Manuel Gonzalo REMACHE- } \\
\text { BUNCI }\end{array}$ & Enfoque cuantitativo & $\begin{array}{l}375 \quad \text { estudiantes } \\
\text { universitarios de II y VII } \\
\text { Ciclo. }\end{array}$ \\
\hline 04 & Colombia & 2018 & $\begin{array}{l}\text { Diego, SALAZAR BLANDÓN } \\
\text { Beatriz, OSPINA RAVE }\end{array}$ & Cuantitativo & $\begin{array}{l}\text { Estudiantes de I y X Ciclos, } \\
\text { pregrado en Enfermería, } \\
\text { Universidad en Medellín, } \\
\text { Colombia, } 2018 .\end{array}$ \\
\hline 05 & Perú & 2019 & $\begin{array}{l}\text { Aníbal Teobaldo VERGARA } \\
\text { VÁSQUEZ }\end{array}$ & Cuantitativo & $\begin{array}{l}60 \text { estudiantes del I Ciclo } \\
\text { de Derecho de la } \\
\text { Universidad Privada } \\
\text { Antenor Orrego, Piura, } \\
2019\end{array}$ \\
\hline 06 & Perú & 2020 & Felicitas, RONDAN ZAMATA & Cuantitativo & $\begin{array}{l}99 \text { estudiantes del I Ciclo } \\
\text { de Psicología, semestre } \\
\text { 2019-II. }\end{array}$ \\
\hline
\end{tabular}

Fuente: elaboración propia 
Al revisar estas investigaciones nuestro trabajo se centró en examinar y comparar las dimensiones, el metodología, conclusiones y discusión de los trabajos de investigación seleccionados. Finalmente preciso mis conclusiones.

\section{Desarrollo y Discusión}

\section{Dimensiones}

Empezaremos examinando las particularidades y luego aquellas diferencias entre estos trabajos de investigación. La primera particularidad respecto al uso de dimensiones lo hace Remache, M. (2018) quien utiliza para su estudio solo dos dimensiones: dimensión sustantiva y dimensión dialógica. Otra particularidad posee Azurín, V. (2018), esta investigadora aplica la lectura crítica, habilidades cognitivas, tipos de razonamiento y habilidades comunicativas como dimensiones para desarrollar pensamiento crítico en el curso de Comunicación. Finalmente, la investigación de Rondan, F. (2020) es particular tambien ya que aplicó lógica criterial y pragmática, para identificar el pensamiento crítico en estudiantes universitarios. Existen coincidencias en las investigaciones de Salazar, D. y Ospina, B. (2018), Vergara, A. (2019) y Lara, V., Ávila, J. y Olivares, S. (2010), han utilizado practicamente las mismas dimensiones, Análisis, Inferencia e Interpretación. Haremos ahora una presentación general de estas investigaciones; De estas seis investigaciones, tres de ellas consideran la dimensión Interpretación en la medición del pensamiento crítico. Lara, V., Ávila, J. y Olivares, S. (2010); Salazar, D. y Ospina, B. (2018 y Vergara, A. (2019) y definiéndola como comprender y expresar el significado o la relevancia de una amplia variedad de experiencias, situaciones, datos, eventos, juicios, convenciones, creencias, reglas, procedimientos o criterios. Esto se da principalmente mediante el parafraseo (Facione, 2007, p. 4). Azurín, V. (2018) es la única que utiliza la Taxonomía de Bloom para dimensionar el pensamiento crítico; estas dimensiones son: Conocimiento, comprensión, aplicación, análisis, síntesis y evaluación. Inferencia, de Priestley, M. (1996, p.25) y Autorregulación, nuevamente la extrae de Facione (2007, p.6). Remache, M. (2018), de Montoya (2007, p.78) utiliza las dimensiones Dialógica y Sustantiva, dimensiones muy conocidas y utilizadas actualmente. Rondan, F. (2020), utiliza las dimensiones de Robert Ennis, Lógica, criterial y pragmática.

Una de las particularidades que hemos encontrado en la investigación de Lara, V., Ávila, J. y Olivares, S. (2010), es el uso de la estrategia Aprendizaje Basado en Problemas 
para desarrollar pensamiento crítico, en el cual a partir de presentar un problema, se plantean posibles soluciones; los estudiantes se autoregulan y guiados por el tutor construyen sus conocimientos como un aprendizaje independiente (Arends, 2007, p. 384.).

Cuando se intenta desarrollar el pensamiento crítico en los estudiantes universitarios, los investigadores utilizan diversas dimensiones; estas diferencias tienen su explicación porque enseñan diversas materias y se establecen objetivos diversos.

\section{Metodología}

Empezamos precisando las coincidencias, todas las investigaciones examinadas, han aplicado el enfoque cuantitativo; en tal sentido, Remache, M. (2018), Azurín, V. (2018), Rondan, F. (2020), Salazar, D. y Ospina, B. (2018), Vergara, A. (2019) y Lara, V., Ávila, J. y Olivares, S. (2010) han utilizado instrumentos para recabar sus datos y procesarlos.

Existe una particularidad en cuanto al método, Lara, V., Ávila, J. y Olivares, S. (2010), fué la única investigación que ha utilizado el enfoque mixto, cuantitativo y cualitativo; para el primero usó un instrumento tipo cuestionario en base a Likert y para el Segundo un instrumento tipo rúbrica. Otra particularidad lo encontramos en la investigación de Azurín, V. (2018), que aplicó investigación cuasi experimental, es decir, dos grupos: uno experimental y otro de control, se les aplicó el pretest de redacción de textos argumentativos. El uso de la metodología es vital en toda investigación, cada investigador decide la metodología que le permita tener mejores resultados.

Ahora presentamos de manera general la metodología que aplicaron las investigaciones examinadas; al revisar las investigaciones de Lara, V., Ávila, J. y Olivares, S. (2010), Azurín, V. (2018), Remache, M. (2018), Salazar, D. y Ospina, B. (2018), Vergara, A. (2019) y Rondan, F. (2020), se puede observar que existe un denominador común respecto a la metodología usada, todas han utilizado el enfoque cuantitativo; este enfoque según Hernández, Fernández y Baptista (2015, p.4.) precisan que este enfoque utiliza la recolección de datos para probar hipótesis con base en la medición numérica y el análisis estadístico, con el fin de establecer pautas de comportamiento y probar teorías. Tres de estas investigaciones han utilizado el estudio cuasi experimental, Lara, V., Ávila, J. y Olivares, S. (2010) y Azurín, V. (2018). El estudio cuasi experimental, es aquel que manipula al menos una variable de investigación de manera deliberada para ver el efecto o relación con una o más variables (Valderrama, 
2010, p.57). Se puede precisar entonces que otra de las similitudes en estas investigaciones es que todas han utilizado cuestionarios para extraer sus datos, aunque cada quien con sus respectivas dimensiones e indicadores y diferentes cantidad de items. Para garantizar la confiabilidad de estos instrumentos, las investigaciones que han utilizado el Coeficiente de Alpha de Cronvach son Lara, V., Ávila, J. y Olivares, S. (2010), Azurín, V. (2018), Remache, M. (2018), Salazar, D. y Ospina, B. (2018) y Rondan, F. (2020). Una distinción que merece mencionarse es la investigación de Lara, V., Ávila, J. y Olivares, S. (2010) respecto al que utilizaron un instrumento cuantitativo tipo cuestionario y uno cualitativo tipo rúbrica. De estas seis investigaciones puede notarse que la población investigada es universitaria de diferentes especialidades y a cantidades; Salazar, D. y Ospina, B. (2018), investigaron a 50 estudiantes de ingeniería médica del VII y VIII Ciclo; Azurín, V. (2018), investigó a 229 estudiantes de la Facultad de Ciencias Sociales y Humanidades; Remache, M. (2018) investigó a 375 estudiantes de dos universidades de I y II Ciclo; Salazar, D. y Ospina, B. (2018) investigaron a 104 estudiantes universitarios del I y X ciclo en Enfermería; Vergara, A. (2019) investigó a 60 estudiantes universitarios del I ciclo de Filosofía; Rondan, F. (2020) investigó a 99 estudiantes universitarios del I Ciclo de Psicología.

\section{Conclusiones}

Al revisar las conclusiones de estas investigaciones, Remache, M. (2018), Azurín, V. (2018), Rondan, F. (2020), Salazar, D. y Ospina, B. (2018), Vergara, A. (2019) y Lara, V., Ávila, J. y Olivares, S. (2010), encontramos que existen algunas coincidencias que a continuación presentamos, todas concluyen que al aplicar sus instrumentos mejoran el desarrollo de pensamiento crítico. 
Cuadro 2. Comparación de Conclusiones

\begin{tabular}{|c|c|c|c|c|}
\hline $\mathrm{N}^{\circ}$ & PAIS & AÑO & AUTOR(ES) & CONCLUSIONES \\
\hline 01 & $\mathrm{a}$ & 2010 & $\begin{array}{l}\text { Verónica LARA } \\
\text { QUINTERO } \\
\text { José Enrique AVILA } \\
\text { PALET } \\
\text { Silvia } \\
\text { OLIVARES } \\
\text { OLIVARES }\end{array}$ & $\begin{array}{l}\text { Una eficaz estategia para desarrollar el } \\
\text { pensamiento crítico es el Aprendizaje } \\
\text { Basado en Problemas (ABP). Promueve } \\
\text { competencias para una formación integral } \\
\text { para la práctica profesional. Esta } \\
\text { competencia se manifiesta en la toma de } \\
\text { desiciones que logran resultados positivos. }\end{array}$ \\
\hline 02 & Perú & 2018 & $\begin{array}{l}\text { Vilma Urbana } \\
\text { AZURÍN } \\
\text { CASTILLO }\end{array}$ & $\begin{array}{l}\text { Cuando un estudiante desarrolla } \\
\text { pensamiento crítico, tiene la capacidad de } \\
\text { estructurar y redactar textos argumentativos. } \\
\text { Eso lo evidencian los resultados (con un } \\
\text { valor de } \mathrm{z}=-2,878 \text { y nivel de significancia } \\
\mathrm{p}=0,004<\alpha=0,05 \text { ) }\end{array}$ \\
\hline 03 & Ecuador & 2018 & $\begin{array}{l}\text { Manuel Gonzalo } \\
\text { REMACHE-BUNCI }\end{array}$ & $\begin{array}{l}\text { Sus resultados demuestran que al aplicar los } \\
\text { instrumentos se desarrolla el pensamiento } \\
\text { crítico en ambas dimensiones: Sustantivas y } \\
\text { dialógicas. }\end{array}$ \\
\hline 04 & Colombia & 2018 & $\begin{array}{l}\text { Diego, SALAZAR } \\
\text { BLANDÓN } \\
\text { Beatriz, OSPINA } \\
\text { RAVE }\end{array}$ & $\begin{array}{l}\text { Las estrategias utilizadas para desarrollar el } \\
\text { pensamientio crítico, son importantes en el } \\
\text { proceso enseñanza aprendizaje, sobre todo en } \\
\text { la capacidad analítica. }\end{array}$ \\
\hline 05 & Perú & 2019 & $\begin{array}{l}\text { Aníbal Teobaldo } \\
\text { VERGARA } \\
\text { VÁSQUEZ }\end{array}$ & $\begin{array}{l}\text { El programa PERIPATO, permite organizar } \\
\text { y desarrollar de manera significativa los } \\
\text { aprendizajes en todas las asignaturas. }\end{array}$ \\
\hline 06 & Perú & 2020 & $\begin{array}{l}\text { Felicitas, RONDAN } \\
\text { ZAMATA }\end{array}$ & $\begin{array}{l}\text { Al aplicar las estrategias para el desarrollo } \\
\text { del pensamiento crítico, per mite que el } \\
\text { estudiante obtenga un aprendizaje } \\
\text { significativo que perdura en el tiempo. }\end{array}$ \\
\hline
\end{tabular}


Discusión

Al comparar la discusión de los trabajos de investigación examinados procederemos a informar lo que, con relación a la investigación de Vergara, A. (2019), se hace la discusión comparando sus resultados con la investigación de Aranda (2014) quien en su tesis titulada "Piensápolis" para el desarrollo del pensamiento crítico tiene resultados parecidos. Tambien hace una comparación con la tesis de Guevara (2016) titulada "Pensamiento Crítico y su relación con el desempeño docente en la Universidad Nacional Mayor de San Marcos, 2015, en cuyo resultado relaciona de manera directa al desarrollo del Pensamientio Crítico con el Desempeño Docente.

Salazar, D. y Ospina, B. (2018), en su artículo científico titulado "Nivel de pensamiento crítico de estudiantes de primero y ultimo semestre de pre grado en enfermería de una Universidad en Medellín, Colombia", hace su discusión precisando que no se encontró significativas diferencias entre el desarrollo del pensamiento crítico de varones y mujeres, en comparación con la investigación de Salahshoor y Rafiee (2015) en Iran, que tiene los mismos resultados.

Respecto a la discusión que hace Rondan, F. (2020), en su investigación doctoral concluye que al aplicar sus instrumentos para el desarrollo del pensamiento crítico es estadísticamente signififativo. Este resultado coincide con los resultados de la investigación de Rojas (2018) que al aplicar sus estrategias obtiene un resultado de nivel medio predominante.

En la discusión de Lara, V., Ávila, J. y Olivares, S. (2010) demuestra que la estrategia de Aprendizaje Basado en Problemas (ABP) es eficaz para el desarrollo del pensamiento crítico, compara sus resultados con Ladouceur y Cols (2004, citado en Olivares y Heredia, 2012), quienes sostiene que esta estrategia desarrollan competencias tales como pensamiento crítico, autodirección, trabajo en equipo, etc. Tambien asocial esta valoración con lo mencionado por Cabrero, Loreto y Peña (2008) quienes sostienen que $\mathrm{ABP}$ es una estrategia que permite al estudiante la apropiación del conocimiento y el desarrollo de otras capacidades profesionales.

\section{Conclusión}

Habiendo examinado estas seis investigaciones nacionales e internacionales relacionadas con el desarrollo del pensamiento crítico en estudiantes universitarios, podemos 
mencionar que existen coincidencias en todas ellas; Todas han utilizado el enfoque cuantitativo, por lo tanto han aplicado instrumentos específicos para extraer datos y realizar la hermenéutica específica. Asimismo, en todas las conclusiones se puede observar que las estrategias utilizadas producen un significativo desarrollo del pensamiento crítico. Respecto a las diferencias se ha podido indentificar que Lara, V., Ávila, J. y Olivares, S. (2010) ha utilizado un enfoque mixto utilizando un instrumento cuantitativo tipo cuestionario y un instrumento cualitativo tipo rúbrica. Otra diferencia significativa presenta Azurín, V. (2018), utilizando el método cuasiexperimental en su investigación; separó a los estudiantes en dos grupos, uno experimental y otro de control. Me permito sugerir que las próximas investigaciones respecto al desarrollo del pensamiento crítico, deben orientar sus esfuerzos en identificar cuál es la estrategia y dimensiones más eficaces para cada Ciencia Social; Ejemplo, para el caso de filosofía o teología, se propondría las dimensiones de análisis, inferencia, juicio y argumentación.

\section{Referencias}

Arends, R. (2007). Aprender a enseñar. México: Mc Graw Hill.

Azurín, V. (2018). Desarrollo del pensamiento crítico y su efecto en la redacción de textos argumentativos de los estudiantes del cuarto ciclo de la Facultad de Ciencias Sociales y Humanidades de la Universidad Nacional de Educación Enrique Guzmán y Valle, año 2015. Lima-Perú

Campos, A. (2007). Pensamiento crítico. Bogotá: Editorial Delfín S.A.

Hernández, R., Fernández, C. y Baptista M. (2015). Metodología de la Investigación. Sexta

edición. México: Mac Graw Hill.

Lara, V.; Ávila, J. y Olivares, S. (2010). Desarrollo del pensamiento crítico mediante la aplicación del Aprendizaje Basado en Problemas, Bogotá, Colombia.

Mahmood Shumalia (2017) Testing the effectiveness of a critical thinking skill intervention for

initial teacher education students in Pakistan. (Tesis inédita de doctorado). Universidad de Southampton, Reino Unido.

Ministerio de Educación (2006). Guía para el desarrollo del pensamiento crítico. Lima: MINEDU. 
Ossa, C.; Palma, M.; Lagos, N.; Quintana, I. y Díaz, C. (2016). Análisis de instrumentos de

medición del pensamiento crítico. Unidad de investigación docente de la Universidad de Concepción de Chile.

Paul, R. y Elder, L. (2005). Estándares de competencia para el pensamiento crítico. Fundación para el Pensamiento Crítico.

Remache, M. (2018). Las dimensiones sustantivas y dialógicas del pensamiento crítico en

estudiantes de bachillerato y universitarios. Universidad Central de Ecuador, Ecuador.

Reverter, J. y Hernández, V. (2012). Artículos científicos: Tipos, Secciones y publicación scientific paper. Grupo de investigación Moviment Huma. Universidad de Lleiva,

Rivas, S. y Saiz, C. ( ) Validación y propiedades psicométricas de pensamiento crítico.

Universidad de Salamanca, España.

Rondan, F. (2020). Estrategias didácticas, desarrollo del pensamiento crítico y su incidencia

en el aprendizaje significativo en estudiantes de una universidad privada, Lima, 2020.

Universidad Cécar Vallejo, Lima, Perú.

Salahshoor N, Rafiee M. The relationship between Critical Thinking and gender: A case of

Iranian EFL learners. J Appl Linguist Lang Res [Internet]. 2016;3(2):117-23. Available from: http://www.jallr.com/index.php/JALLR/article/viewFile/219/pdf219

Salazar, D. y Ospina, B. (2018). Nivel de pensamiento crítico de estudiantes de primero $\mathrm{y}$

último semestre de pre grado en enfermería de una universidad de Medellín,

Colombia.

Valderrama, S. (2010). Pasos para elaborar proyectos y tesis de investigación científica. Lima: San Marcos.

Vergara, A. (2019). Programa peripato para desarrollar el pensamiento crítico de los estudiantes del primer ciclo de derecho de la Universidad Privada Antenor Orrego, Campus Piura- 2019, Perú.

Villarini, A. (2003). Teoría y pedagogía del pensamiento crítico. Universidad de Puerto Rico. 